\title{
EL MINI VIDEO COMO RECURSO DIDÁCTICO EN EL APRENDIZAJE DE MATERIAS CUANTITATIVAS
}

\author{
(MINI VIDEOS AS DIDACTIC RESOURCES IN QUANTITATIVE SUBJECTS)
}

\author{
Damián de la Fuente Sánchez \\ Montserrat Hernández Solís \\ Inmaculada Pra Martos \\ Universidad Nacional de Educación a Distancia, UNED (España)
}

\section{RESUMEN}

La utilización de herramientas audiovisuales con fines educativos se ha generalizado en las últimas décadas, siendo el vídeo una de las más destacables. Sin embargo, su utilización en la enseñanza de materias de carácter cuantitativo no está tan extendida como en otras. A partir de nuestra experiencia docente de aplicación de vídeos de corta duración en la enseñanza a distancia de la matemática financiera, hemos extraído una serie de conclusiones que presentamos en este trabajo y que se refieren a los principios básicos que consideramos debe reunir el vídeo para conseguir unos resultados óptimos de aprendizaje por parte de los estudiantes. También damos a conocer la opinión de los estudiantes sobre la utilidad que les ha proporcionado este tipo de herramienta con unos resultados que ponen de manifiesto la utilidad de esta herramienta en la enseñanza a distancia de materias cuantitativas.

Palabras clave: mini videos, videos educativos, educación a distancia.

\begin{abstract}
The usage of audiovisual tools for educational purposes has become widespread in recent decades-with videos becoming one of the most prominent. However, its usage for the teaching of subjects of a quantitative nature is not as extensive as for other subjects. Based on our teaching experience of applying short videos in the distance teaching of financial mathematics, we were able to arrive at a series of conclusions. These refer to the basic principles that a short video should consist of, so as to achieve optimal learning results by students. We also present the students' opinions about the usage of these tools - which is illustrated with some findings that support their usage in the distance/online teaching of quantitative subjects.
\end{abstract}

Keywords: short videos, educational video, distance education. 
La educación a distancia no ha sido ajena al desarrollo de las tecnologías de la información y la comunicación (TIC) en los últimos años. Aunque los textos y guías de estudio siguen constituyendo el material didáctico básico en este tipo de enseñanza, se han ido implantando progresivamente otros recursos de aprendizaje de carácter más tecnológico que han permitido una transición eficaz de la clase presencial a la clase virtual. A través de las plataformas virtuales se han ido reuniendo todos esos recursos (foros, chats, autoevaluaciones, cuestionarios, audios, vídeos,...) que permiten reforzar y reorientar las acciones formativas mediante un proceso flexible, rápido y eficaz, además de posibilitar los espacios de intercambio y comunicación en acciones formativas, tal como señalan Imbernón, Silva y Guzmám (2011).

También la enseñanza presencial ha ido incorporando en su metodología de aprendizaje los avances experimentados en el uso de las TIC. En el caso de las materias cuantitativas de tipo económico, aunque la mayoría de los profesores siguen confiando en las clases magistrales a la hora de desarrollar el temario (Becker y Watts, 1996; Becker, 1997), no menos cierto es que con el paso del tiempo se han ido introduciendo paulatinamente otros recursos didácticos, tales como discusiones en clase, clips de películas para ilustrar diversos conceptos económicos (Leet y House, 2003; Mateer y Li, 2008; Rodríguez, 2011; Sexton, 2006), vídeos o audios (Siegfied et al., 1991), edición de libros especiales que conjugan economía y literatura (Watts, 2003), vídeos de proyectos de estudiantes (Holtzblatt y Tschakart, 2011), ilustraciones de novelas (Vachis y Bohanan, 2012), novelas (Cotti y Johnson, 2012), música (Tinari y Khandke, 2000) e incluso espectáculos y series de televisión (Hall, 2005; Lucasen y Thomas, 2010; Mateer et al., 2011).

En el campo de la matemática financiera se ha apostado, desde hace algunos años, por adaptar algunos de estos recursos didácticos con un doble objetivo: por un lado, ayudar a los estudiantes a mejorar el aprendizaje de los conceptos y desarrollos matemáticos más complicados y, por otro lado, disminuir la tasa de fracaso que históricamente se aprecia en esta disciplina.

En este artículo se considera, en primer lugar, el papel del vídeo como herramienta al servicio de la educación y el aprendizaje y la orientación didáctica que se le ha dado en esta experiencia. En segundo lugar, se define al mini vídeo como una tecnología específica con unas características propias y cómo hay que preparar y producir un mini vídeo que sirva para mejorar el aprendizaje de materias cuantitativas. En la siguiente sección se comentan las estrategias de uso que deben tener en cuenta los usuarios de esta tecnología y se describe la plataforma que se ha utilizado para elaborar y distribuir estos mini vídeos. Por último, se evalúa de 
una forma muy descriptiva la utilidad que ha supuesto esta herramienta para los estudiantes de la UNED que han hecho uso de ella.

\section{LA FUNCIÓN EDUCATIVA DEL VÍDEO}

Las funciones del vídeo en el contexto educativo dependen de su naturaleza bidireccional y autosuficiencia a la hora de generar mensajes. Así, se puede utilizar como transmisor de información, como medio para aprender, como herramienta de evaluación de aprendizajes y habilidades o como instrumento de investigación. Normalmente su interés didáctico se ha derivado de la integración de distintas características intrínsecas a su naturaleza:

- Fusión de medios audiovisuales que permite llegar a un espectro más amplio de estudiantes y le hace ser preferido frente al libro de texto, tal como se apunta en un reciente estudio de Hedge, Useem y Martínez (2011).

- $\quad$ Posibilidad de ofrecer un feed-back inmediato.

- Flexibilidad en su utilización.

- Control local de la producción.

No obstante lo anterior y de acuerdo con Salomón (1979, 1991), lo relevante del vídeo como elemento de enseñanza tiene que ver más con los sistemas simbólicos que desarrolla, con la interacción cognitiva con el estudiante y cómo se utiliza. En esta experiencia la orientación que se dará al diseño y producción tendrá que ver con su función esencial de instrumento de adquisición de conocimiento, con la idea de aclarar conceptos y realizar una síntesis de ideas esenciales en la materia. No se pretende con este recurso construir el conocimiento en el sentido de que el estudiante aprenda por sí mismo y que así desarrolle destrezas que le permitan resolver problemas, sino simplemente superar el modelo tradicional de transmisión de conocimientos y habilitar un sistema que permita al alumno un aprendizaje autónomo y flexible. Además, se pretende contribuir, tal como afirma un estudio de Corporation for Public Broadcasting (2004), a reforzar el material de lectura, a mejorar la comprensión, a proporcionar una mejor integración de distintos estilos de aprendizaje y a aumentar la motivación y el entusiasmo de los estudiantes. 


\section{EL MINI VIDEO Y SU DISEÑO}

Para aproximar el concepto de mini vídeo, se partirá de las definiciones de vídeo educativo que se encuentra en la literatura sobre medios materiales y recursos curriculares en el campo de la educación. Algunos autores, como Bartolomé (1988), clasifican los medios visuales (vídeo) y auditivo (audio-clases) con el criterio de presentación del código y otros, como Area (2004), incluyen el vídeo dentro de los medios icónicos y las audio-clases dentro de los medios auditivos. Pascual (2011), por su parte, define el vídeo educativo como un medio didáctico que facilita el desarrollo de un proceso de aprendizaje y destaca los cuatro componentes que lo conforman:

- Un soporte material.

- Un contenido.

- Una forma simbólica de representar la información.

- Una finalidad o propósito educativo.

Las definiciones anteriores son válidas también para los mini videos, con algunas matizaciones. Las principales diferencias entre ambas herramientas se refieren tanto a la duración como al enfoque adoptado en cada uno de los casos. En cuanto al primero de los aspectos mencionados hay que tener en cuenta que esta es una de las variables más importantes puesto que se trata de condensar en un breve espacio de tiempo una definición o un concepto determinado. Por lo que respecta al enfoque didáctico se trata de conseguir que el estudiante sea un sujeto activo en el proceso de aprendizaje y no un mero receptor pasivo.

De acuerdo con lo señalado anteriormente, se puede definir al mini vídeo como un vídeo de corta duración que constituye un material didáctico de tipo tecnológico para transmitir una determinada información que ayude a consolidar cierto aprendizaje. Estableciendo un paralelismo con los cuatro elementos del vídeo que señala Pascual (2011), se distinguen los siguientes componentes en el mini vídeo educativo aplicado al aprendizaje de materias cuantitativas:

- El soporte material es el vídeo que se realiza en el entorno de un aula AVIP (aulas virtuales creadas en la UNED con la tecnología IP). 
- El segundo de los componentes (el contenido), lo integran los conceptos, demostraciones matemáticas y casos reales propios de la materia objeto de estudio.

- La forma simbólica de representar la información es el aparato matemático propio de la materia, mediante presentaciones en formato PowerPoint.

- Por último, la finalidad o propósito educativo es reforzar el proceso de aprendizaje de aquellos conceptos que, a lo largo de la experiencia como docentes, se ha detectado que son los que presentan más dificultades en su comprensión y asimilación por parte de los estudiantes.

En el proceso de elaboración de un mini vídeo y, siendo coherentes con la naturaleza que se le ha atribuido, sería necesario seguir las fases que distintos especialistas en la materia han señalado como indispensables en el diseño de un medio para la enseñanza y el aprendizaje y que ha de tener en cuenta diversas variables comunicativas y pedagógicas.

Son varios los trabajos de investigación dedicados al análisis de las fases que han de seguirse en el proceso de elaboración de un vídeo para la enseñanza y el aprendizaje. En todos ellos se contemplan etapas bien diferenciadas y en cada una de ellas se tienen en cuenta diversas variables comunicativas y pedagógicas. Así, Martínez (2004) apunta al diseño como la primera fase en la elaboración de un vídeo, estableciendo los contenidos y la relación entre ellos, su secuenciación y la forma de presentación. La segunda fase es la producción, en la que se materializa el diseño utilizando los recursos y conocimientos técnicos apropiados. Previamente a estas dos fases, habrá tenido lugar una fase de diagnóstico del problema a tratar y que justificaría las acciones posteriores.

Otros autores, como Cabero (1994) consideran tres fases: diseño, producción y posproducción y evaluación. La fase de diseño se concreta, a su vez, en diversas subetapas:

- Análisis de la situación, que comprende desde la selección del tema y los contenidos sobre los que se tratará el material hasta la determinación del equipo técnico necesario.

- Planificación y temporalización del proceso de desarrollo. 
- La documentación, que debería incluir no sólo la información conceptual sino alguna referencia visual o auditiva.

- Y por último, la guionización de la información.

Simultáneamente a esta tarea se tomará la decisión de elegir el modelo de producto que se quiera obtener que, en este caso y siguiendo a Sigüenza (2004), se trataría de un producto a medida ya que está diseñado por los propios profesores y con un objetivo muy concreto.

Siguiendo algunas de las pautas señaladas anteriormente, se han elaborado los mini vídeos en varias fases. En la fase del diseño se han considerado los siguientes aspectos:

- Selección de los contenidos o temas a explicar que, en la experiencia como docentes en la materia durante veinte años, se han detectado como más difíciles de entender por los estudiantes o aquellos que son básicos.

- Identificación del grupo al que irá destinado. En esta experiencia el grupo objetivo potencial lo constituyen todos los estudiantes matriculados en la asignatura durante el curso académico.

- Determinación de los medios técnicos a utilizar. Aunque la elaboración de un mini vídeo se puede hacer con unas transparencias y una simple cámara de vídeo, en esta experiencia se ha contado también con la tecnología audiovisual de última generación que ha desarrollado la UNED a través de las denominadas aulas AVIP.

- Temporalización del proceso u ordenación de las diferentes actividades que se van a llevar a cabo para elaborar cada mini video:

- Identificación de los conceptos a tratar.

- Preparación del guión.

- Producción de las transparencias necesarias.

- Ensayo de la presentación.

Dentro de este esquema de trabajo hay dos cuestiones que requieren especial atención en la fase de diseño: la guionización y la elaboración de las transparencias. 
La primera de ellas comienza con la preparación de una sinopsis que recoja los objetivos del mini vídeo y los contenidos a desarrollar en el mismo. En esta fase es importante tener en cuenta algunas pautas generales de carácter didáctico, en la línea señalada por Pascual Sevillano (2011):

- El tiempo de duración no conviene que sea superior a 5 minutos, coincidente con la opinión de Stone (1999) o de Sexton (2006). Si al diseñar el vídeo se sobrepasa ese tiempo, lo más prudente es dividirlo en varios, porque diversos estudios como el de Eliis y Childs (1999), demuestran que los estudiantes pierden gran parte de su interés a partir de duraciones superiores a 10 minutos.

- Es muy conveniente introducir elementos tanto simbólicos como narrativos.

- Es necesario que al final del mini vídeo haya un breve sumario en el que se resuman las principales ideas tratadas.

- Es importante recordar, en todo caso, que debe prevalecer el aspecto didáctico sobre el técnico.

- Es muy recomendable la utilización de gráficos como recurso didáctico.

La segunda cuestión a la que hay que prestar especial atención es el soporte sobre el que se realizan los mini vídeos. Se trata de transparencias, normalmente elaboradas en PowerPoint y trasladadas a formato pdf. Esas transparencias deben basarse en una filosofía simple, que se podría calificar como minimalista, en las que se sugiere a través de una frase, un gráfico o una ecuación la posterior explicación que se va a desarrollar. Se trata de establecer una base prácticamente en blanco sobre la que se desarrollen los conceptos a analizar en cada mini-vídeo (figura 1) que luego podrán descargar los propios estudiantes y que les pueden servir como elemento para recordar y reflexionar sobre lo aprendido. De forma esquemática las características más importantes de las transparencias que se han manejado son las siguientes:

- Menos de cuatro o cinco transparencias por mini-vídeo de 5 minutos de duración.

- Realizadas en PowerPoint y posteriormente editadas en formato pdf.

- Se ha utilizado un tipo de letra generosa en su tamaño (mínimo 14 ppp).

- No se han incluido ni número de página ni secciones.

- Se ha dejado un hueco para insertar la ventana de la cámara web. 


\section{DESCUENTO DE PAPEL COMERCIAL}

\section{Definición}

\section{Una operación financiera simple a través de la cual una entidad financiera entrega al cliente el valor actual de un capital futuro que se representa mediante un efecto de comercio.}

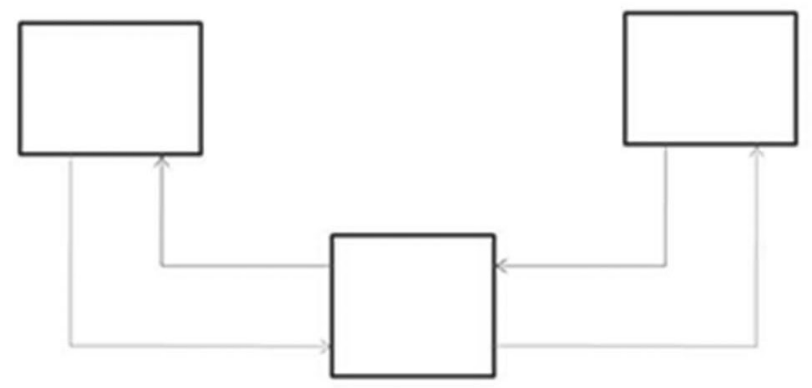

Figura 1.

En cuanto a la fase de producción, se trata de llevar a cabo las acciones planificadas en el diseño junto con la grabación de las imágenes. En el caso de los mini videos no tiene sentido hablar de fase de posproducción, ya que no es posible seleccionar, ordenar o acoplar las imágenes una vez grabado el mini-vídeo. Para salvar, en la medida de lo posible, este inconveniente, es importante ser muy cuidadoso con la elaboración del guión y en el ensayo previo a la fase de grabación real.

Por último, y en la línea sugerida por Pascual Sevillano (op.cit) es aconsejable realizar una guía didáctica en la que se aclaren los requerimientos técnicos que deben tener los equipos para su visionado y sugerencias para su utilización didáctica. Esta guía debe poseer una estructura en la que se especifiquen los objetivos que persigue el material, un breve resumen del contenido presentado, el nivel aconsejable de utilización y una propuesta de actividades a realizar con los estudiantes. 


\section{ESTRATEGIAS DE UTILIZACIÓN Y TECNOLOGÍA EMPLEADA}

Para conseguir el aprovechamiento óptimo de las posibilidades de aprendizaje de este medio se plantea una estrategia de utilización basado en las recomendaciones de Cabero, Llorente y Román (2005).

Antes del visionado es preciso contextualizar el vídeo en el capítulo correspondiente del temario, explicando por qué se centra en uno u otro concepto y los objetivos que se persiguen. También convendría planificar las actividades de extensión a realizar por los estudiantes después del visionado, tales como una batería de preguntas a las que deberían contestarse.

Durante el visionado. En una enseñanza presencial el profesor podría, en el aula, utilizar las posibilidades instrumentales del vídeo (pausa, avance rápido, retroceso,...) para realizar intervenciones didácticas. En la educación a distancia no se desprecian esas posibilidades pero el papel del profesor se transforma ligeramente a través de una intervención virtual asíncrona.

Después del visionado. Las actividades finales enunciadas en la primera etapa deben tener como objetivo central contrastar la calidad y cantidad de la información proporcionada, así como la profundización en la misma a través de la realización de diferentes trabajos, debates en grupo vía foros, búsqueda de ejemplos en un contexto real, etc.

Aunque se pueden realizar mini vídeos utilizando una pizarra digital convencional y una grabadora de vídeo corriente, se ha optado por utilizar la tecnología que la UNED ha dispuesto en el marco de las aulas AVIP.

Durante los últimos años la Universidad Nacional de Educación a Distancia, UNED, ha venido desarrollando una nueva estructura tecnológica ajustada a sus necesidades, tanto de sus enseñanzas como de los aprendizajes de los estudiantes a distancia, en el marco de los centros asociados (plan ATECA). La denominada conferencia en línea es una de las posibilidades de comunicación audio-visual por internet (AVIP) que actualmente puede utilizarse. Aunque está diseñada para atender las necesidades de tutorización en remoto de los estudiantes de la UNED de forma sincrónica y a partir de la estructura multisede de esta universidad, se ha aprovechado para elaborar mini-vídeos que, una vez alojados en un repositorio, permiten al estudiante interesado descargarlos y verlos cuantas veces quieran, sin más limitaciones que el ancho de banda que dispongan en su conexión por internet. Se utiliza así lo que se denomina Cadena Campus que sería un segundo nivel de 
servicio de las aulas AVIP y que básicamente consiste en la captura de sesiones emitidas desde aulas AVIP o desde casa (si se dispone de una conexión a internet, una tableta digitalizadora y una cámara web) para su almacenamiento y difusión en directo y diferido por internet.

De esta forma se utiliza la posibilidad asíncrona de la herramienta que, aunque impide la interrelación en tiempo real con el estudiante, habilita para conseguir el objetivo fundamental propuesto con la elaboración de este material: disponer de un recurso didáctico audio-visual que ayude al estudiante a estudiar, asimilar y aprender los conceptos e ideas básicas de la materia.

\section{EVALUACIÓN DE LA HERRAMIENTA}

Aunque parece indiscutible el valor positivo de la utilización de diversas herramientas multimedia en el aprendizaje de materias científicas, en general, y de la matemática financiera, en particular, se ha querido contrastar esa opinión con el juicio de los estudiantes que han tenido la oportunidad de acceder a ellas a lo largo de su estudio de esta asignatura en los cursos académicos 2010-2011 y 2011-2012.

Enelprimerañodeimplantación delos mini-vídeos comoherramienta multimedia se elaboró una encuesta que se distribuyó entre los estudiantes matriculados en la asignatura Matemática Financiera I correspondiente a la Diplomatura en Ciencias Empresariales y a la Licenciatura en Administración y Dirección de Empresas y que se presentaron a la correspondiente prueba presencial.

La muestra fue bastante representativa puesto que del total de estudiantes matriculados (en torno a 1.500) se trabajó con 446 encuestas. Con las respuestas recibidas se trató de analizar si había habido algún tipo de influencia positiva entre el uso de las herramientas multimedia y la calificación obtenida en la prueba presencial a través de un análisis estadístico de dependencia lineal y que no reveló una dependencia significativa. Lo que sí es relevante es la opinión que mostraron los encuestados sobre la herramienta de los mini-vídeos y las conferencias en línea y que se resume en los siguientes aspectos:

El 52\% y el 47\% de los encuestados consultaron los mini-vídeos y las conferencias en línea, respectivamente.

El 85\% de los estudiantes que consultaron los mini-vídeos y las conferencias en línea los consideraron didácticos. 
En cuanto a la utilidad que les han proporcionado a los estudiantes estas herramientas los resultados son bastantes satisfactorios puesto que casi el 66\% de los que utilizaron los mini-vídeos y las conferencias en línea les calificaron como útil o muy útil.

El 84\% de los encuestados coincidieron en señalar que estas herramientas deben aplicarse no sólo a los conceptos teóricos sino a la parte práctica de la materia.

En el curso 2011-2012 se cambió la metodología de la valoración de las herramientas en dos sentidos: en primer lugar, fueron consultados los estudiantes de la asignatura Matemática Financiera correspondientes al Grado en Administración y Dirección de Empresas y, en segundo lugar, se realizó una encuesta en línea a través del curso virtual a los estudiantes matriculados (y no a los que se examinaron en la prueba presencial) para valorar con carácter general todas las herramientas de aprendizaje y consulta alojados en dicho curso virtual (figura 2). Los resultados muestran bastante satisfacción por parte de los estudiantes en casi todos los ítems, muy en la línea de otros estudios similares, como por ejemplo, el de Cabero, Llorente y Puentes (2010).

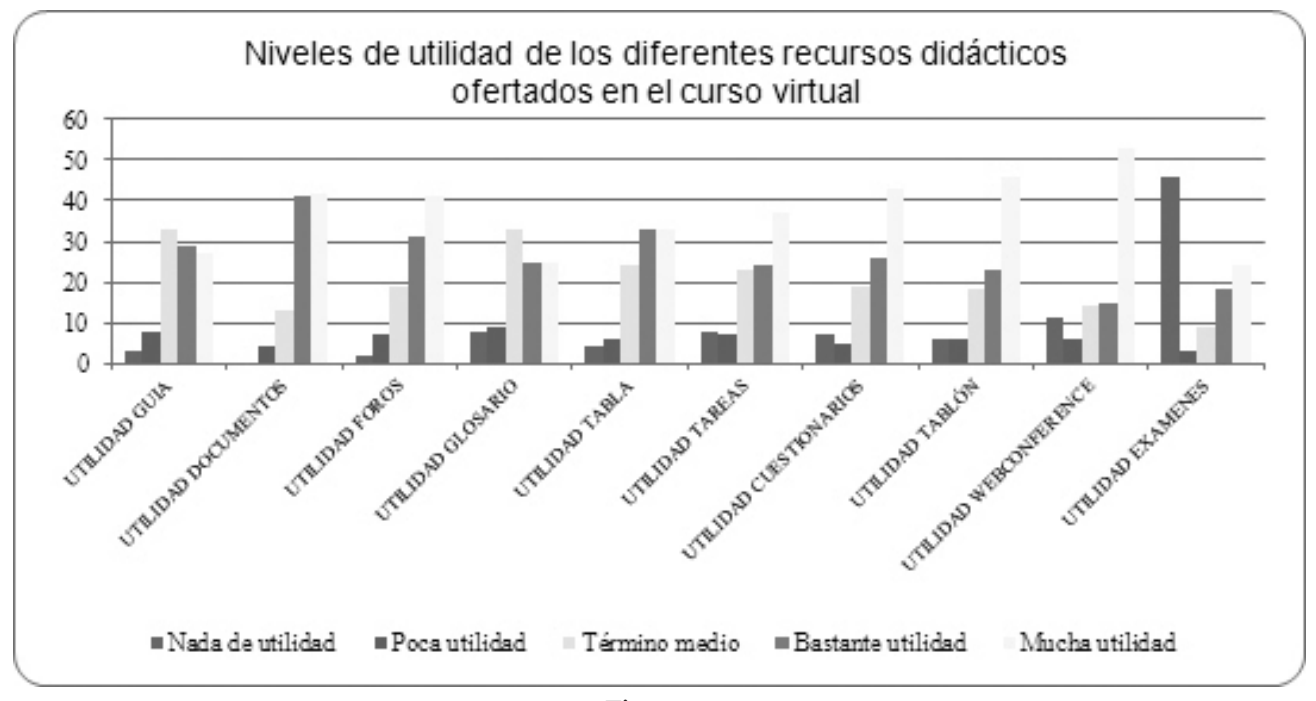

Figura 2.

En el caso particular de los mini-vídeos mejoró ligeramente la opinión que los estudiantes mostraron sobre la utilidad que les proporcionó esta herramienta a la hora de reforzar el aprendizaje de la matemática financiera. Concretamente, un $63 \%$ de los encuestados respondieron que el uso de los mini-vídeos les había 
proporcionado mucha o bastante utilidad (figura 3) y el 68\% respondieron con la misma calificación, si la pregunta se refería a las conferencias vía web, muy en la línea de diversos estudios empíricos sobre la utilidad del vídeo con carácter general, tales como los de Hilgenberg y Tolone (2000), Zhang (2005), Choi y Johnson (2005), o sobre los efectos que produce en el aprendizaje de la matemática (Boster et al., 2007). Por otro lado, y al igual que ocurrió en el curso anterior, casi todos los encuestados señalaron que estas herramientas deberían aplicarse al tratamiento de casos prácticos y no sólo de tipo teórico como se ha hecho hasta ahora.

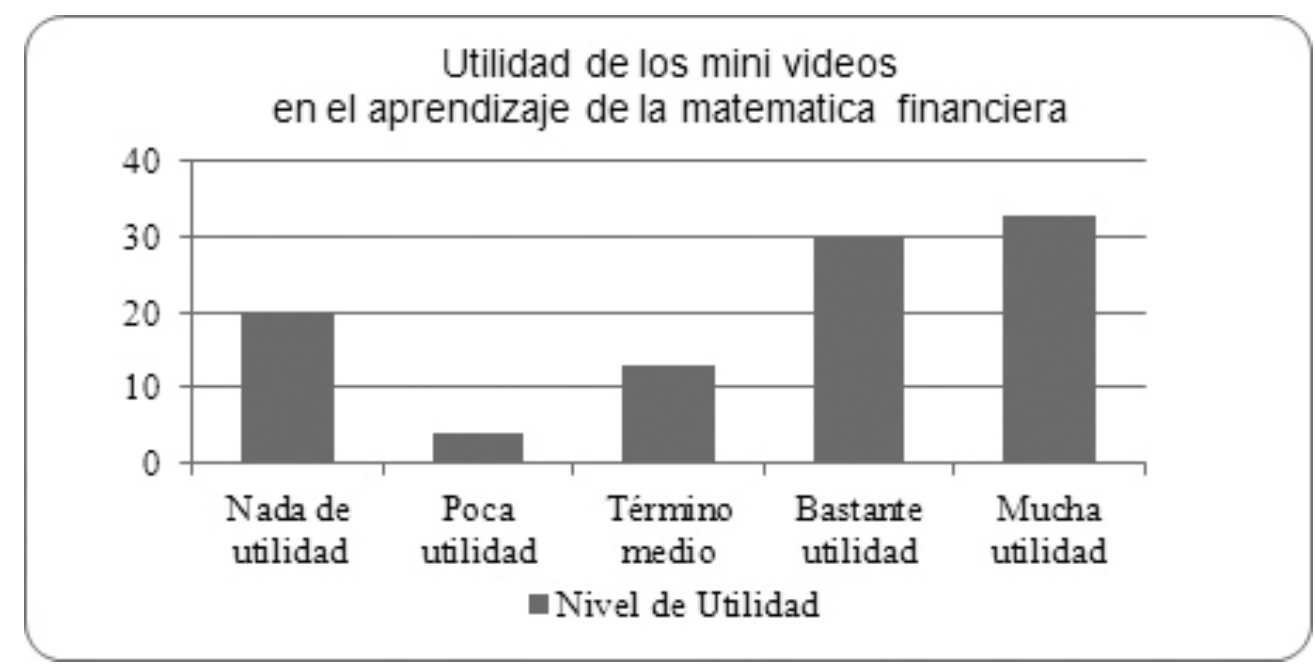

Figura 3 .

Curiosamente contrastan estas opiniones con la escasa dependencia que encontramos en el curso 2010-2011 entre la utilización de este recurso didáctico y las calificaciones finales obtenidas por los estudiantes que la usaron, en la línea que señalan otros autores, tales como Agarwal y Day (1998) o Savage (2009).

\section{CONCLUSIONES}

El uso de las nuevas tecnologías, en general, y del vídeo, en particular, es indiscutible a la hora de hacer más atractivo el aprendizaje y la enseñanza en el siglo $\mathrm{XXI}^{1}$. La idea que subyace detrás de esta forma de enseñanza en la que se incorpora el vídeo es que el estudiante pueda ver las explicaciones tantas veces como quiera en su casa, rebobinar, detenerse o ir hacia delante. En la enseñanza presencial los estudiantes podrán compartir lo aprendido, resolver dudas y desarrollar una interacción más activa con el profesor. En la enseñanza a distancia se traslada el 
aula al salón de casa, acabando así con una de las limitaciones de esta modalidad educativa, y se ayuda a que el estudiante despeje sus dudas.

Pero no vale cualquier vídeo. Una tecnología mal aprovechada en la que se desprecia el diseño y en la que el talento comunicador brilla por su ausencia provocará, inevitablemente, rechazo entre los destinatarios. Para ello es fundamental cumplir con las pautas que se han desarrollado en este trabajo y que podemos agrupar de la siguiente forma:

- Para qué se va a utilizar el vídeo.

- Cómo se va a hacer el vídeo.

- De qué manera se va a implantar el vídeo.

La experiencia al aplicar estas herramientas audiovisuales de corta duración en el campo de la matemática financiera ha permitido comprobar las ventajas que conlleva su utilización. Entre ellas cabe destacar, en primer lugar, la de facilitar la asimilación de contenidos y ecuaciones que presentan mayor dificultad para el estudiante y, en segundo lugar, la de hacer más sencilla la comprensión de una materia que con los métodos de enseñanza tradicionales puede resultar bastante laborioso. Por todo ello, parece que el esfuerzo realizado en el desarrollo de casi 30 mini-vídeos ha sido positivo y los resultados obtenidos en las encuestas realizadas entre los estudiantes que utilizaron este recurso didáctico así lo confirman. No obstante, se pretende seguir profundizando en esta experiencia didáctica en los próximos años a través de la ampliación del catálogo con nuevas propuestas y se tratará de valorar de forma cuantitativa la incidencia del uso de este tipo de medios en la evaluación final.

\section{NOTAS}

1. Proyectos como la academia Khan (http://www.khanacademy.org/) o iniciativas como las del profesor Walter Lewin (http://web.mit.edu/physics/people/faculty/ lewin walter.html) o de Juan Medina (http://www.lasmatematicas.es) así lo certifican. 


\section{REFERENCIAS BIBLIOGRÁFICAS}

Area, M. (2004). Los medios y la tecnología en la educación. Madrid: Pirámide.

Agarwal, R.; Day, A. (1998). The impact of the Internet on economic education. The Journal of Economic Education, 29 (Spring), (99-110). DOI: 10.1080/00220489809597973.

Bartolomé, A. (1988). Concepción de la tecnología educativa a finales de los ochenta. Biblioteca virtual de Tecnología educativa. [en línea] Disponible en: http:// tecnologiaedu.us.es/bibliovir/pdf/bartolo1.pdf [consulta 2012, 11 de noviembre].

Becker, W. E. (1997). Teaching economics to undergraduates. The Journal of Economic Education, 35, (1347-1373).

Becker, W. E.; Watts, M. (1996). Chalk and talk: A national survey on teaching undergraduates economics. American Economic Review, 86, (448-453).

Boster, F. J.; Meyer, G. S. et al. (2007). The impact of video streaming on mathematics performance. Communication Education, 56 (2), (134-144). DOI: 10.1080/03634520601071801.

Cabero, J. (1994). Evaluar para mejorar: medios y materiales de enseñanza. In Sancho, J. Para una tecnología educativa. Barcelona. Horsori.

Cabero, J.; Llorente, C.; Román, P. (2005). Las posibilidades del vídeo digital para la formación. Labor Docente, 4, (58-74).

Cabero, J.; Llorente, C.; Puentes, A. (2010). La satisfacción de los estudiantes en red en la formación semipresencial. Comunicar, 35, (149-157). DOI: 10.3916/C352010-03-08.

Choi, H. J.; Johnson, S. D. (2005). The Effect of Context-Based Video Instruction on Learning and Motivation in Online Courses. American Journal of Distance Education, 19 (4), (215-227). DOI: 10.1207/ s15389286ajde1904_3.

Corporation for Public Broadcasting. (2004). Television goes to school: The impact of video on student learning in formal education. [en línea] Disponible en: http:// www.dcmp.org/caai/nadh173.pdf [consulta 2012, 05 de mayo].

Cotti, C.; Marianne, J. (2012). Teaching economics using historical novels: Jonathan Harr's The Lost Painting. The Journal of Economic Education, 43, (3), (269-281). DOI: $10.1080 / 00220485.2012 .686391$.

Ellis, R.; Childs, M. (1999). The effectiveness of video as a learning tool in online multimedia modules. Journal of Educational Media, 24 (3), (217-223). DOI: 10.1080/1358165990240305.

Hall, J. (2005). Homer economicus: using The Simpsons to teach economics. Journal of Private Enterprise, 30, (165-176).

Hedge, S.; Useem, A.; Martínez, S. (2011). Engaging with business learning: the source and medium do matter. [en línea] Disponible en: http://assets.bigthink. com/WhitePaper1.pdf [consulta 2012, o3 de marzo].

Hilgenberg, C.; Tolone, W. (2000). Student perceptions of satisfaction and opportunities for critical thinking in distance education by interactive video. American Journal of Distance Education, 14 (3), (59-73). DOI: 10.1080/08923640009527065.

Holtzblatt, M.; Tschakart, N. (2011). Expanding your accounting classroom with digital video technology. Journal of Accounting Education, 29, (100-121). DOI: 10.1016/j.jaccedu.2011.10.003.

Imbernón, F.; Silva, P.; Guzmán, C. (2011). Competencias en los procesos de enseñanza-aprendizaje virtual y semipresencial. Comunicar, 36, (107-114). DOI: 10.3916/C36-2011-03-01

Leet, D.; Houser, S. (2003). Economics goes to Hollywood: Using classic films and documentaries to create an undergraduate economics course. The Journal of Economic Education, 34, (326-332). DOI: $10.1080 / 00220480309595226$. 
Luccasen, R. A.; Thomas, M. K. (2010). Simpsonomics: Teaching economic using episodes of The Simpsons. The Journal of Economic Education, 41 (2), (136-149). DOI: $10.1080 / 00220481003613847$.

Martínez, F. (2004). Bases generales para el diseño, la producción y la evaluación de medios para la formación. En Salinas, J. A. Tecnologías para la educación. Madrid: Alianza.

Mateer, G. D.; Li, H. (2008). Movie scenes for economics. The Journal of Economic Education, 39, 303. DOI: 10.3200/ JECE.39.3.303.

Mateer, G. D.; Ghent, L. S.; Stone, M. (2011). $\mathrm{Tv}$ for economics. The Journal of Economic Education, 42 (2), 207. DOI: 10.1080/00220485.2010.548229.

Pascual, M. A. (2011). Principios pedagógicos en el diseño y producción de nuevos medios, recursos y tecnologías. En Sevillano, M. L (Coord.). Medios, recursos didácticos y tecnología educativa. Madrid: Pearson Educación.

Rodriguez, C. (2011). Capitalism in six westerns by John Ford. The Journal of Economic Education, 42 (2), (191-194). DOI: 10.1080/00220485.2011.555721.

Salomon, G. (1979). Interaction of media, cognition and learning. Londres: Jessey Bass.

Salomon, G. (1981). Communication and education: an interactional approach. Los Angeles: Sage.

Savage, S. (2009). The Effect of Information Technology on Economic Education. The Journal of Economic Education, 40 (4), (337-353). DOI: 10.1080/00220480903237901.
Sexton, R. L. (2006). Using Short Movie and Television Clips in the Economics Principles Class. The Journal of Economic Education, 37 (4), (406-417). DOI: 10.3200/JECE.37.4.406-417.

Siegfried, J. R.; Bartlett, W.; al (1991). The status and prospects of economics majors. The Journal of Economic Education, 22, (197-224).

Sigüenza, J. (2004). Diseño de materiales docentes multimedia en entornos virtuales de enseñanza-aprendizaje. [en línea] Disponible en: http://www.ucm.es/ info/multidoc/multidoc/revista/num8/ siguenza.html [consulta 2012, 03 de diciembre].

Stone, L. (1999). Multimedia Instruction Methods. The Journal of Economic Education, 30 (3), (265-275). DOI: $10.1080 / 00220489909595987$.

Tinari, F. D.; Khandke, K. (2000). From Rhytm and blues to Broadway: using music to teach economics. The Journal of Economic Education, 31 (3), (253-270). DOI: $10.1080 / 00220480009596784$.

Vachis, M. A.; Bohanan, C. E. (2012). Using illustrations from American novels to teach about labor market. The Journal of Economic Education, 42 (1), (72-82). DOI: 10.1080/00220485.2012.636712.

Watts, M. (2003). The literary book of economics. Wilmington, DE: ISI.

Zhang, D. (2005). Interactive MultimediaBased E-Learning: A Study of Effectiveness. American Journal of Distance Education, 19 (3), (149-162). DOI: 10.1207/s15389286ajde1903_3. 


\title{
PERFIL ACADÉMICO Y PROFESIONAL DE LOS AUTORES
}

Damián de la Fuente Sánchez. UNED, Dpto. de Economía de la Empresa y Contabilidad, Facultad de Ciencias Económicas y Empresariales, Profesor Titular de Universidad.

E-mail: dfuente@cee.uned.es

Montserrat Hernández Solís. Dpto. de Economía de la Empresa y Contabilidad, Facultad de Ciencias Económicas y Empresariales de la UNED, Profesora Ayudante.

E-mail: montserrath@cee.uned.es

Inmaculada Pra Martos. UNED, Dpto. de Economía de la Empresa y Contabilidad, Facultad de Ciencias Económicas y Empresariales, Profesora Colaboradora

E-mail: ipra@cee.uned.es

DIRECCIÓN DE LOS AUTORES

\author{
UNED \\ Dpto. de Economía de la Empresa y Contabilidad \\ Facultad de Ciencia Económicas y Empresariales \\ Paseo Senda del Rey, 11 \\ 28040, Madrid, España
}

Fechas de recepción del artículo: 01/03/13

Fecha de aceptación del artículo: 14/05/13

\section{Como citar este artículo:}

Fuente Sánchez, D. de la; Hernández Solís, M.; Pra Martos, I. (2013). El mini video como recurso didáctico en el aprendizaje de materias cuantitativas. RIED. Revista Iberoamericana de Educación a Distancia, volumen 16, nº 2, pp. 177-192. 